\title{
Demonstration of Dynamic Medium Access Control for APON and SuperPON
}

\author{
E. Ringoot, N. Janssens, M. Tassent, J. Angeloupoulos ${ }^{*}$, C. Blondia ${ }^{* *}$, P. Vetter
}

Alcatel Research \& Innovation, F. Wellesplein 1, B-2018 Antwerp

*National Technical University of Athens (NTUA)

**University Institutes of Antwerp (UIA)

\begin{abstract}
A dynamic MAC (Medium Access Control) protocol demonstrator for ATM PON (Passive Optical Network) was realised and tested. The prototype is based on a SuperPON transport system, which allows implementing downstream grants per ONT (Optical Network Termination) or subdivided per T-Cont class (Transfer Container) and upstream requests by piggybacking or dedicated polling slots. The demonstrator platform was made configurable in order to evaluate dynamic bandwidth allocation schemes for SuperPON and for G.983.dba APON.
\end{abstract}

\section{INTRODUCTION}

Although APON (ATM Passive Optical Network) systems were already tested in field trials in the mid nineties [1], it is since the approval of the ITU-T G.983.1 [2] standard in 1998 and the availability of low cost EO (Electro-Optical) transceivers that APON has gained significant momentum. Today APON is generally recognised as the most suited technology for FTTH (Fibre To The Home). The recent endorsement of G.983.3 (formerly "G.983.wdm") enables the implementation of an overlay service by WDM (Wavelength Division Multiplexing), such as video distribution, which is an attractive added value to voice and data services over APON for residential subscribers.

The present paper reports on the demonstration of dynamic MAC (Medium Access Control) protocols, which allow for a more efficient use of the upstream bandwidth in a PON than by static MAC protocols. Dynamic Bandwidth Allocation schemes for APON are currently under discussion by the FSAN group (Full Service Access Network) for standardisation in G.983.dba [3].

Two types of TDMA (Time Division Multiple Access) PON are considered in this paper:

- $\quad$ The commercially available standard APON, which features a downstream bitrate of 622 or $155 \mathrm{Mbit} / \mathrm{s}$ and an upstream bitrate of $155 \mathrm{Mbit} / \mathrm{s}$ shared by up to 64 ONT (Optical Network Termination) at a maximum distance of 10 or $20 \mathrm{~km}$ from the central office, ${ }^{1}$

1 The realisation of the MAC controller and the study of dynamic MAC protocols for APON are partially funded by the Flemish IWT project "ITA2-FTTH".
- The long term SuperPON, which features a downstream bitrate of $2.5 \mathrm{Gbit} / \mathrm{s}$ and an upstream bitrate of $311 \mathrm{Mbit} / \mathrm{s}$ shared by 2048 ONT at a maximum range of $100 \mathrm{~km}$ [4],[5]. ${ }^{2}$

The MAC demonstrator is based on the more performing SuperPON transport system, but was made configurable to allow the evaluation of dynamic bandwidth allocation in standard APON as well. Since many subscribers are connected to a single line termination of a SuperPON, DBA (Dynamic Bandwidth Allocation) was recognised as an important way to gain in throughput by statistical multiplexing [6]. The transport system enabling chips were therefore provided with features that enable dynamic MAC protocols [7]. The MAC components presented in [8] are now integrated in the system and tested. Several papers with simulations on DBA have been published [6]. To the authors' knowledge, it is however the first time that a real hardware demonstration of such a dynamic MAC for ATM PON is achieved.

\section{DyNAMIC MAC PROTOCOL AND CHANNELS}

In a shared medium, such as the PON, in which the upstream bandwidth from the ONT to the LT (Line Termination) is shared by a TDMA mechanism, a MAC controller at the LT is required to arbitrate the access for each timeslot. In an APON, the timeslot is an ATM cell. There are two main methods for implementing a centrally controlled MAC Protocol. The first method allocates the bandwidth to an ONT by generating a fixed rate of permits (also called grants) for that ONT based on signalling information. This is called a rate-based generator and is also often referred to as a static MAC protocol. A second option is to generate permits at the LT, based on the requests generated by the ONT and transmitted via a polling or piggyback channel. This is often referred to as a dynamic MAC. It is clear that the dynamic MAC will be much more efficient for bursty data traffic, as compared to the static MAC. If an efficient cell scheduling and buffering mechanism is used at the ONT side, it is already shown by simulations that dynamic MAC can be very efficient for transporting bursty TCP/IP traffic.

\footnotetext{
${ }^{2}$ The study of the MAC protocols for SuperPON and the realisation of the ACRA chip are partially funded by the European ACTS project
} PELICAN. 


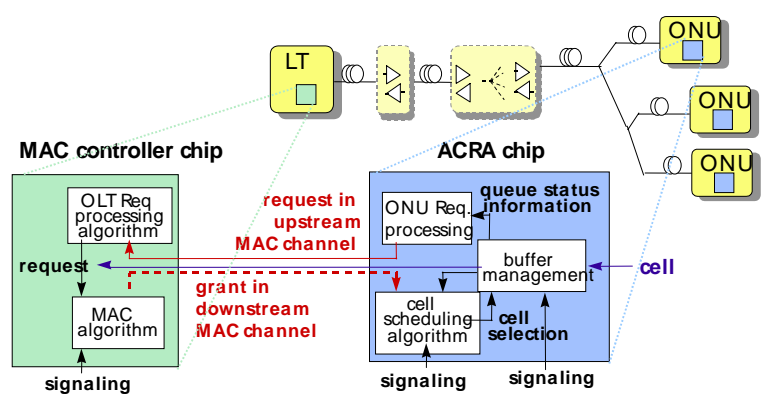

Figure 1: Basic operation of the MAC protocol.

The operation of a MAC protocol requires the transmission of a grant from LT to ONT, which indicates the ONT that is allowed to use an upstream time slot. It is even possible to make a subdivision of grants per QoS (Quality of Service) category T-Cont (Transmission Container) in an ONT. The latter is sometimes referred to as coloured grants. To improve the bandwidth efficiency for some types of T-Cont, it is beneficial to report the number of data cells in the queues at the ONT that are waiting for a permission to be transmitted in the upstream direction. This is called the request channel of the MAC.

The grants (also called permits) generated by the MAC Controller are encapsulated in the PON transport system in dedicated fields of the PLOAM cells (Physical Layer Operation And Maintenance) (cf. Figure 2). While a grant field of 8 bits is defined in a G.983.1 APON, a field of 16 bits was implemented in the present demonstrator to accommodate a large number of ONT connected to one SuperPON LT and/or distinguish different T-Conts (coloured grants).

Two types of request channels were implemented: dedicated divided slot cells (also called RAU (Request Access Unit), which are sent per group of ONTs in socalled multi-burst slots at regular polling intervals, and a piggyback field, which is 1 byte in the 3 bytes preamble of each data cell. The divided slot allows that one upstream timeslot (56 bytes) is used by 8 consecutive ONTs to report one mini-slot each (consisting of 3 byte PLP (Physical Layer Preamble), 3 bytes request data, and 1 byte CRC (Cyclic Redundancy Check)). The piggyback MAC channel is implemented as one byte overhead in the 3-byte preamble of each upstream timeslot. It allows an ONT to request new permits while sending an upstream cell. Both channels could be implemented while remaining compliant with the G.983.1 format. The divided slot approach is considered sufficient for a standard APON. The piggyback approach is an additional way to report requests in a PON with large splitting factors, because the interval between divided slots for a specific ONT becomes too long.

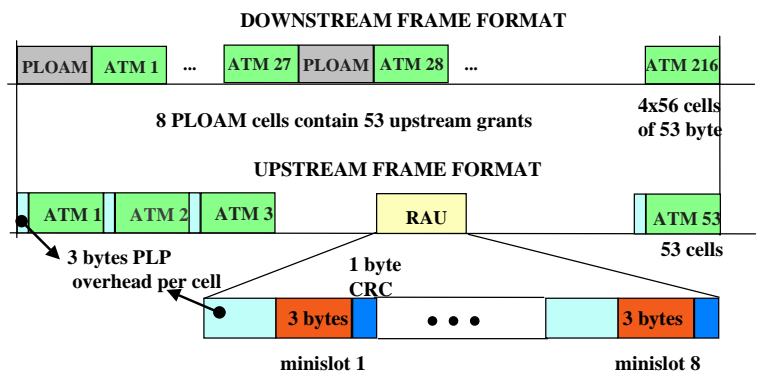

Figure 2: Frame format: Downstream PLOAM cells contain grant fields - Upstream minislots and PLP (Physical Layer Preamble) contain requests.

\section{MAC BUILDING BLOCKS}

The implementation of the dynamic MAC functionality on the network required the introduction of a dynamic MAC chip-set, as shown in Figure 1. On the LT side a MAC controller chip was introduced while every ONT contains an ACRA (ATM Cell Routing and Adaptation) chip.

\section{A. The LT MAC controller chip}

Connections from an ONT to the LT with similar service guarantees can be grouped in a MAC Transfer Container (T-Cont) type. The MAC controller supports 4 T-Cont types per ONT. The implementation of the TCont types differs in the way permits are generated and the priority the generated permits will obtain for forwarding to the ONTs. The architecture of the MAC Controller consists of a permit multiplexing module to schedule all pending permits in static priority order and a generic permit scheduler module for every T-Cont and ONT (Figure 3).

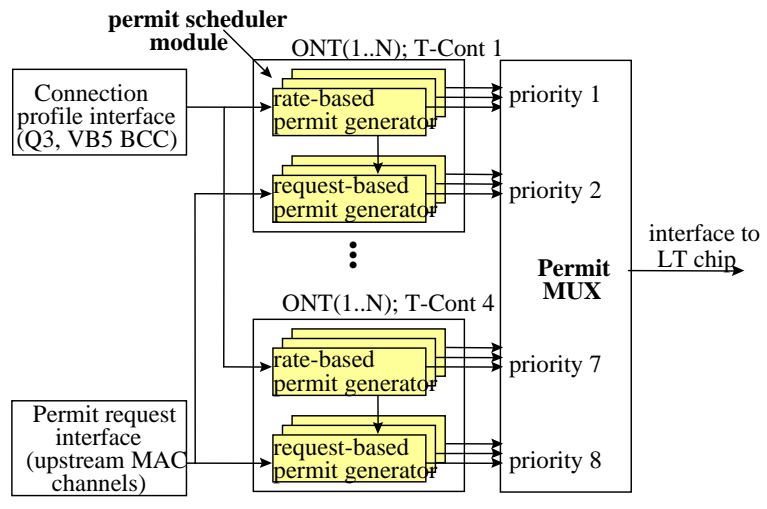

Figure 3: MAC Controller architecture at LT.

The permit scheduler modules consist of a rate-based and a request-based permit generator. The rate-based permit generator enables the generation of pre-arbitrated permits according to a programmable rate. The requestbased permit generator allows the generation of permits explicitly requested by the ONTs. Depending on the TCont class, permits are generated rate based only, request 
based only or both request based and rate based with two different burst level detection alternatives.

Within a specific T-Cont, eligible permits targeting different ONTs are serviced according to a workconserving WRR (Weighted Round Robin) scheduling discipline in the Permit MUX.

\section{B. The ONT ACRA chip}

The ACRA chip at the ONT receives the grant from the SPENT. The scheduler decides about the queue to be emptied, thereby taking into account the priorities and service level agreements. In the case of coloured grants, only the queue(s) of a specific T-Cont can be selected. The ACRA chip furthermore performs frame aware buffer acceptance mechanisms and translates the queue status into the proper request information (e.g. increase of queue length or total number of cells waiting).

The ACRA is conceived as a generic multi-QoS ATM switching element. It consists of a MAC processing module, an ATM traffic switch and a number of Access Points (AP). The ATM traffic switch and the APs are interconnected through a bi-directional ATM internal bus (Figure 4) which is structured in timeslots.

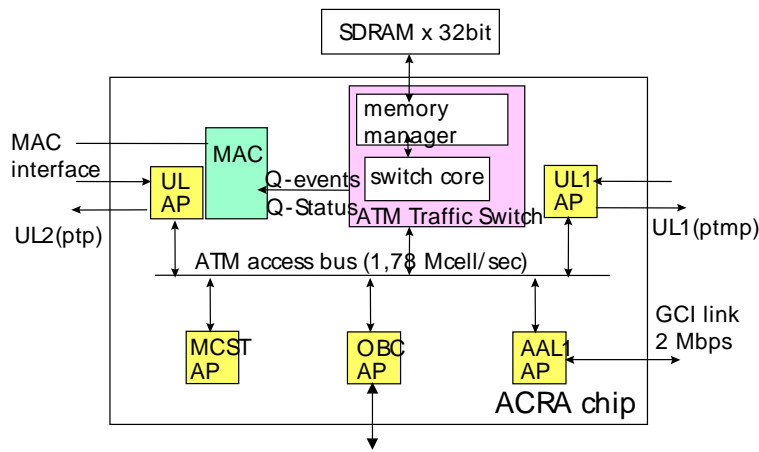

Figure 4: ACRA architecture at NT.

Basically, the ACRA is a store-and-forward system. Cells received on the APs are 'uploaded' into a centralised queuing system under control of the ATM traffic switch. These cells remain in this queuing system until they are 'downloaded' by one of the attached access points. For the network and user AP arriving cells are first processed by a Look Up Table unit to determine the queue destination and header translation (if any). The MAC module pre-processes download requests before forwarding them to the traffic switch for further evaluation.

The MAC module implements cell scheduling by accepting the permits with preferred QoS class information. The module investigates whether there is any active connection corresponding to this class which has a waiting cell. If so, a WRR mechanism determines which of the connections corresponding to this service class is serviced for the corresponding upstream timeslot.
If no such connection exists, programmable priority jumping allows fetching and sending a cell from a different service class and as such is work conserving.

The buffer management in the ACRA switch divides the buffer space in up to 127 FIFO queues and determines whether a cell will be down- or uploaded. The protocol works on a cell by cell base but is generic to also support packet awareness. As such (partial) packet discard mechanisms can be used to enhance upstream link efficiency even further.

Every cell up- or download is reported to the MAC module. This enables the MAC module to determine the number of waiting cells per service class at any moment. In case a data permit arrives at the ACRA the MAC request processing unit will report the number of newly arrived cells per service class since the previous reporting. Four service classes are reported in round robin or fixed priority order. For RAU (polling) permits the $\mathrm{MAC}$ request processing unit will report the total number of cells waiting for all $4 \mathrm{~T}$-Cont simultaneously.

\section{RESULTS}

All required building blocks for the DBA demonstrator were implemented and integrated in the SuperPON platform. A Burst Level Traffic Generator /Analyser board (from the company 4plus) was used for analysis.

First tests to evaluate the dynamic system behaviour have been made by allocating nearly all upstream bandwidth to 'background' ONTs, while leaving only limited bandwidth available for the ONT under test. For the ONT under test three T-Conts have been configured:

T-Cont 1: Rate based permit allocation at $2 \mathrm{Mbit} / \mathrm{s}$, plus burst level setting for request based permits equal to 4 ,

T-Cont 2: Request based permit allocation only,

T-Cont 3: Request based permit allocation only (best effort).

A $4^{\text {th }}$ T-CONT would be the highest priority fixed rate CBR type, but was not considered in the present tests because it does not participate in the dynamic MAC protocol.

For T-Cont 1 the rate based permit generator in the T-Cont module will generate $2 \mathrm{Mbit} / \mathrm{s}$ equivalent permits which are forwarded to the ONT. If the actual traffic for this ONT/T-Cont combination is less than $2 \mathrm{Mbit} / \mathrm{s}$, than no excess bandwidth is generated. If the requested bandwidth is (temporarily) higher the ONT will report requests equivalent to more than $2 \mathrm{Mbit} / \mathrm{s}$. They are stored in the request based generator until the burst level detection mechanism detects that the rate based permit generator can not serve the pending request alone, i.e. the number of pending requests will become higher than 
4. If this is detected the request-based generator will generate the additional permits.

The PON delay was set to 150 upstream timeslots (216 $\mu$ s), which corresponds to a range of $20 \mathrm{~km}$. A polling permit was sent once every 100 upstream timeslots (i.e. one polling every $144 \mu$ s). Different groups of tests were carried out focusing in different characteristics of the system. Two results will be described here.

\section{A. Delay versus average load}

In this scenario the system behaviour (cell transfer delay) is observed under various load conditions, but for the same tagged source traffic. Both sources injected TCont class 1 traffic during this test.

The tagged source traffic is characterised by VBR behaviour of $80 \mathrm{Mbit} / \mathrm{s}$ peak, $40 \mathrm{Mbit} / \mathrm{s}$ average, for various burst size (from 20, to 200, to 2000) so as to evaluate the queue reaction to this traffic. When the burst size is 2000 , the measured values are given in.

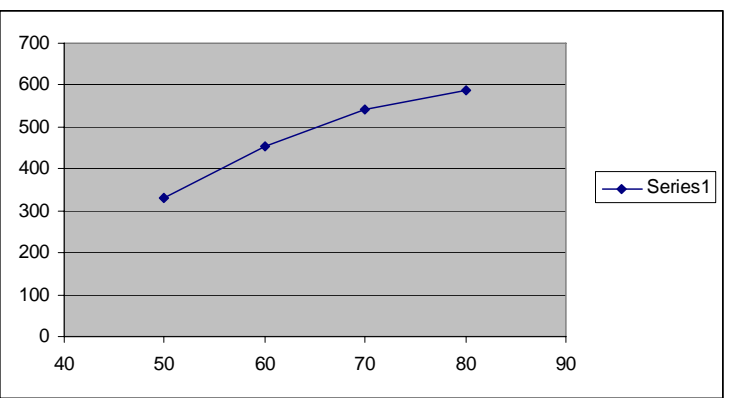

Figure 5: Cell delay in $\mu$ s versus total load (in \%) for burst size 2000.

The delay values that the two sources experienced under the same total load and burst size are nearly the same, as expected since they both use T-Cont class 1 . The delay increases when the total system load increases. In case of $50 \%$ system load, the mean delay observed was $330.39 \mu$ s while in case of $80 \%$ load the mean delay was $587.34 \mu \mathrm{s}$.

\section{B. Impact of priorities}

A second test evaluated the impact of priorities on the traffic. Each source is related to one T-Cont class, served by the MAC Controller. This scenario was performed for average system load (namely 80\%). The rates of the sources as well as the priority of the established connections are listed in the table below.

As shown in, the two connections of the same T-Cont class (1-2, 3-4, 5-6) experience similar performance and close delay values although the rates are different, (more precisely the $2^{\text {nd }}$ source's rate is double the first one's and so on for the two other pairs). The performance is independent of the source rate as expected. It depends on the T-Cont class used.

T-Cont class 3 traffic suffers the system load as it observes the higher delay values. The first two T-Cont classes achieve better performance, as it was designed to be in order to support real time applications. The mean transmission delay for the first two T-Cont classes is almost similar. The difference between these classes is the maximum delay value they experience, resulting in different delay variation. The first one, which is meant to provide the highest QoS supported in the system, achieves not only low mean delay value but also limited delay variation, due to the "burst level" based mechanism. The $2^{\text {nd }}$ one achieves low mean delay but the variation is higher than the first one, while the last one experiences high delay values and is very well suited to best effort services, such as IP applications that could benefit from the flow control mechanism of the TCP protocol.

Table 1: Impact of priorities on traffic delay

\begin{tabular}{|l|l|l|l|l|l|l|l|}
\hline Source & $\begin{array}{l}\text { T-Cont } \\
\text { Class }\end{array}$ & VCI & $\begin{array}{l}\text { PCR } \\
\text { (Mbit/s) }\end{array}$ & $\begin{array}{l}\text { MCR } \\
\text { (Mbit/s) }\end{array}$ & MinCTD & MeanCTD & MaxCTD \\
\hline 1 & $1(\mathrm{CBR})$ & 21 & 5 & 5 & 44.90 us & 292.08 us & 829.30 us \\
\hline 2 & $1(\mathrm{CBR})$ & 22 & 10 & 10 & 44.10 us & 294.14 us & 848.30 us \\
\hline 3 & $2(\mathrm{VBR})$ & 23 & 35 & 17,75 & 44.50 us & 275.47 us & $1,117.20$ us \\
\hline 4 & $2(\mathrm{VBR})$ & 24 & 70 & 35 & 44.60 us & 275.97 us & $1,125.90$ us \\
\hline 5 & $3(\mathrm{VBR})$ & 25 & 35 & 17,75 & $59,395.20$ us & $76,831.96$ us & $97,735.30$ us \\
\hline 6 & $3(\mathrm{VBR})$ & 26 & 70 & 35 & $59,333.10$ us & $76,859.06$ us & $97,698.70$ us \\
\hline
\end{tabular}

Note for the reviewers: The above measurement results represent the test status at the time of writing (March $\left.30^{\text {th }}, 2001\right)$, they prove the proper operation of the demonstrator and confirm the behaviour predicted by simulations. It is expected that more measurement results will be available by August 2001, when the camera-ready manuscript is due. The simulation results on the next page will be replaced by additional experimental results. It is expected that the conclusions in the last section will not change. 


\section{Comparison of coloured versus colourless grants}

An interesting experiment is to compare the efficiency of a MAC protocol based on grants per ONT versus grants per T-Cont in an ONT (Coloured). The results in Figure 6 show that there is no advantage in having the additional complexity of coloured grants. The traffic sources used are based on the reference service basket defined in FSAN for the study of G.983.dba.
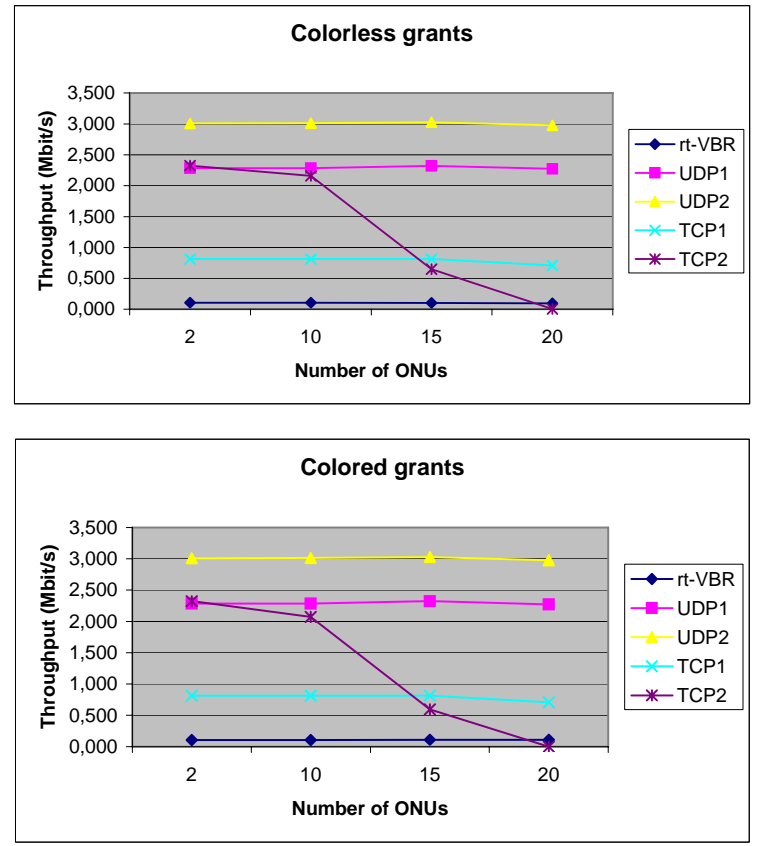

Figure 6: Simulations (and anticipated experimental result by mid 2001) of throughput for coloured versus colourless grants.

\section{SUMMARY AND CONCLUSION}

A MAC protocol demonstrator was realised and tested. The configurable platform supports the following features:

- $\quad$ up to 4 T-Cont types per ONT to group connections with similar QoS guarantees,

- grants per ONT or coloured grants per T-Cont,

- polling of requests in divided slots or in piggybacks,

- setting of typical PON parameters as number of ONT connected, upstream bitrate, and round trip time (range).

It enables the validation of dynamic bandwidth allocation schemes in a standard APON or a long range, high splitting SuperPON. It is a valuable tool to evaluate different proposals under discussion in FSAN for G.983.dba. An important conclusion for APON is that there is no need for the complex implementation of coloured grants.
Compared to the concepts and simulations reported in the past, the present demonstrator proofs the feasibility of dynamic MAC protocols on PON in a physical realisation.

\section{ACKNOWLEDGMENT}

The authors would like to thank all their colleagues and partners in the ITA2-FTTH and PELICAN projects for the valuable contributions to the results.

REFERENCES

[1] G. Baker and G. Van der Plas, "High speed access for the full service network”, EFOC\&N'95, Brighton, 1995.

[2] ITU G. 983.1: "broadband optical access systems based on passive optical networks (PON)".

[3] FSAN-OAN G.983.DBA Draft Recommendation, Feb. 21, 2001.

[4] C. Coene et al, "Uplink performance evaluation of super optical access networks”, ECOC 99, Nice, September 99, pp.138-139.

[5] I. Van de Voorde, C. Martin, J. Vandewege, and X.Z. Qiu, "The SuperPON Demonstrator: an Exploration of Possible Evolution Paths for Optical Access Networks”, IEEE Communications magazine, February 2000, pp.74-82.

[6] R. Hoebeke, K. Venken, and S. Stojanovski, "Dynamic Bandwidth Allocation on SuperPON", SPIE 99, All Optical Networking 1999, Architecture, Control, and Management Issue, September 1999, Boston.

[7] I. Van de Voorde, C. Martin, E. Ringoot, H. Slabbinck, M. Tassent, C. Bouchat, D. Goderis, P. Vetter, X.Z. Qiu, and J. Vandewege "The Super PON demonstrator: a versatile platform to evaluate possible upgrades of the G.983 APON", ISSLS, Stockholm, June 2000.

[8] E. Ringoot, R. Hoebeke, H. Slabbinck and M. Verhaert "Chip-set for quality of service support in passive optical networks", SPIE conference on AllOptical Communication Systems: Architecture, Control and Network Issues, November 98, Boston. 